\title{
De novo domestication of wild tomato using genome editing
}

\author{
Agustin Zsögön ${ }^{1,7}$ (1) , Tomáš Čermák ${ }^{2,6,7}$, Emmanuel Rezende Naves ${ }^{1}$, Marcela Morato Notini ${ }^{3}$, Kai H Edel ${ }^{4}$, \\ Stefan Weinl ${ }^{4}$, Luciano Freschi ${ }^{5}$, Daniel F Voytas ${ }^{2}$, Jörg Kudla ${ }^{4}$ \& \& Lázaro Eustáquio Pereira Peres ${ }^{3}$ (i)
}

\begin{abstract}
Breeding of crops over millennia for yield and productivity ${ }^{1}$ has led to reduced genetic diversity. As a result, beneficial traits of wild species, such as disease resistance and stress tolerance, have been lost ${ }^{2}$. We devised a CRISPR-Cas9 genome engineering strategy to combine agronomically desirable traits with useful traits present in wild lines. We report that editing of six loci that are important for yield and productivity in presentday tomato crop lines enabled de novo domestication of wild Solanum pimpinellifolium. Engineered S. pimpinellifolium morphology was altered, together with the size, number and nutritional value of the fruits. Compared with the wild parent, our engineered lines have a threefold increase in fruit size and a tenfold increase in fruit number. Notably, fruit lycopene accumulation is improved by $500 \%$ compared with the widely cultivated $S$. lycopersicum. Our results pave the way for molecular breeding programs to exploit the genetic diversity present in wild plants.
\end{abstract}

Tomato (S. lycopersicum) is the most important vegetable fruit worldwide, with annual production of 100 million tons ${ }^{3}$. The domestication process from the putative ancestral progenitor, S. pimpinellifolium, which produces pea-sized fruits, to modern tomato varieties is well described ${ }^{4}$. However, despite the increases in yield conferred by domestication, the breeding focus on yield has been accompanied by a loss of genetic diversity and reduced nutritional value and taste ${ }^{5}$.

Many domestication traits have Mendelian inheritance patterns and involve loss-of-function or gain-of function mutations ${ }^{6}$ (Table 1 and Supplementary Table 1). This means that it should be possible to recreate these traits in a suitable genetic background with CRISPR-Cas9 genome editing technology ${ }^{7}$. Although the first CRISPR-Cas9 applications created deletions, modern variants of CRISPR-based genome editing technologies can produce targeted insertions, exchange amino acids and modulate gene expression. Therefore, genome editing could be used to domesticate wild plants and reunite lost but desirable traits, including nutritional features or stress tolerance, with yield potential and other agronomically valuable characteristics ${ }^{8}$.
We previously identified a suite of loci that have shaped the morphology and agronomic potential of current cultivars of tomato, maize, rice and other crops and proposed a reverse genetic approach for the de novo domestication of novel crops $^{9}$ (Table 1 and Supplementary Table 1). In tomato, at least six loci important for key domestication traits have been identified: general plant growth habit (SELFPRUNING $)^{10}$, fruit shape $(\text { OVATE })^{11}$ and size (FASCIATED and FRUIT WEIGHT 2.2)12,13, fruit number (MULTIFLORA) ${ }^{14}$, and nutritional quality (LYCOPENE BETA CYCLASE) ${ }^{15}$. We set out to create a novel crop derived from $S$. pimpinellifolium by targeting this set of genes using a multiplex CRISPR-Cas9 approach to generate loss-of-function alleles. We constructed a single CRISPR-Cas9 plant transformation vector, pTC321 (Supplementary Note 1), which harbored six single guide RNAs (gRNAs) targeting specific sequences in the coding regions of all six genes (Supplementary Fig. 1). Using this vector, we generated ten primary $\mathrm{T}_{0}$ lines, of which three were grown to maturity. $\mathrm{T}_{1}$ seeds were harvested from plant 3 , which showed an oval fruit phenotype, indicative of successful editing of the ovate locus, and determinate growth habit, indicative of loss of function of the selfpruning gene. Sequencing of all six targeted loci in $50 \mathrm{~T}_{1}$ lines revealed that four of the six targeted loci were successfully edited in all 50 lines and harbored indel mutations (Supplementary Tables 2 and $\mathbf{3}$ ). The four edited genes were SELF-PRUNING (SP), OVATE (O), FRUIT WEIGHT 2.2 (FW2.2) and LYCOPENE BETA CYCLASE (CycB). For all four edited genes, we recovered only edited alleles and did not detect any wild-type (WT) alleles in the $\mathrm{T}_{1}$ generation (Supplementary Datasets 1 and 2). However, we did not recover any mutations in either FASCIATED (FAS) or MULTIFLORA (MULT). In the case of FAS we identified a G-to-A substitution in the S. pimpinellifolium genome at the gRNA target site (designed based on the S. lycopersicum genome) and the targeted $S$. pimpinellifolium sequence, which may have prevented editing (Supplementary Tables 2 and $\mathbf{3}$ ).

To address the specificity of our multiplex editing approach, we sequenced the two most closely related off-target loci (as determined by in silico analysis using Geneious R11 program) for each gRNA in two pTC321 plant 3 lines (Supplementary Fig. 2). We did not

\footnotetext{
${ }^{1}$ Departamento de Biologia Vegetal, Universidade Federal de Viçosa, Viçosa, Brazil. 2Department of Genetics, Cell Biology and Development, Center for Genome Engineering, University of Minnesota, Minneapolis, Minnesota, USA. ${ }^{3}$ Departamento de Ciências Biológicas, Escola Superior de Agricultura "Luiz de Queiroz," Universidade de São Paulo, Piracicaba, Brazil. ${ }^{4}$ Institut für Biologie und Biotechnologie der Pflanzen, Universität Münster, Münster, Germany. ${ }^{5}$ Instituto de Biociências, Universidade de São Paulo, São Paulo, Brazil. ${ }^{6}$ Present address: Inari Agriculture, Cambridge, Massachusetts, USA. ${ }^{7}$ These authors contributed equally to this work. Correspondence should be addressed to J.K. (jkudla@uni-muenster.de) or L.E.P.P. (lazaro.peres@usp.br).
} 
Table 1 Known domestication genes in cereal (maize, Zea mays) and non-cereal (soybean, Glycine max) crops

\begin{tabular}{|c|c|c|c|c|c|c|}
\hline Crop species & Gene target & Function & Mutation type & Genetic effect & Phenotypic outcome & Refs. \\
\hline \multirow[t]{4}{*}{ Maize } & $T b 1$ & $\begin{array}{l}\text { TCP-family transcription } \\
\text { factor }\end{array}$ & $\begin{array}{l}\text { Retrotransposon insertion } \\
\text { in regulatory region }\end{array}$ & Gain of function & $\begin{array}{l}\text { Inhibition of side branching, altering source-sink } \\
\text { relations and increasing yield }\end{array}$ & 31,32 \\
\hline & $\lg 1$ & $\begin{array}{l}\text { Squamosa-promoter } \\
\text { binding protein }\end{array}$ & Retrotransposon insertion & Loss of function & Leaf is upright due to absent ligules and auricles & 28,33 \\
\hline & $\operatorname{tga} 1$ & $\begin{array}{l}\text { SBP-box transcription } \\
\text { factor }\end{array}$ & $\begin{array}{l}\text { SNP altering single amino } \\
\text { acid }\end{array}$ & Gain of function & Changes encased to naked kernels & 34 \\
\hline & $Z m C C T$ & $\begin{array}{l}\text { CCT domain-containing } \\
\text { protein }\end{array}$ & $\begin{array}{l}\text { Retrotransposon insertion } \\
\text { in regulatory region }\end{array}$ & Loss of function & Reduction of photoperiod sensitivity & 35,36 \\
\hline \multirow[t]{3}{*}{ Soybean } & DT1 & $\begin{array}{l}\text { CETS family of regulatory } \\
\text { genes }\end{array}$ & SNPs altering amino acids & Loss of function & $\begin{array}{l}\text { Changes growth from indeterminate to determinate, } \\
\text { producing a shorter, more compact plant }\end{array}$ & $37,38^{a}$ \\
\hline & GA200x & $\begin{array}{l}\text { Gibberellin biosynthesis } \\
\text { enzyme }\end{array}$ & Variation in promoter region & Loss of function & Seed weight & 39 \\
\hline & SHAT1-5 & $\begin{array}{l}\text { NAC-family transcription } \\
\text { factor }\end{array}$ & $\begin{array}{l}\text { 20-bp deletion disrupting a } \\
\text { repressive element }\end{array}$ & Gain of function & $\begin{array}{l}\text { Increased secondary wall biosynthesis promoting thick- } \\
\text { ening of fiber cap cells, leading to reduced shattering }\end{array}$ & 40 \\
\hline
\end{tabular}

aActually targets the close ortholog GmFT2.

observe editing of either off-target locus in any of the sequenced lines (Supplementary Dataset 1 ).

Next we examined the phenotypes of two $\mathrm{T}_{2}$ plant lines (designated as line 3-5 and line 3-11). The parental $\mathrm{T}_{1}$ plants of both 3-5 and 3-11 contained homozygous deletions in the second exon of the gene encoding SP (Fig. 1a). Breeding an $s p$ allele with a single nucleotide polymorphism (SNP) in the coding region into tomato cultivars was instrumental in enabling the mechanical harvest of fruits ${ }^{16}$. Similarly, loss of SP function in S. pimpinellifolium resulted in compact plants with reduced height, reduced number of sympodial units and determinate growth when compared with WT S. pimpinellifolium (Fig. 1b-g). In the fruit-shape-determining $\operatorname{OVATE}(O)$ locus, genome editing had induced distinct homozygous deletions in the first exon of the gene in both $\mathrm{T}_{1}$ parents (Fig. 1h). As predicted, loss of $O$ gene function in both alleles caused an oval fruit shape (Fig. 1i-m). The elongated form of fruits occurs through reduced internal fruit pressure and is associated with less rain-induced fruit cracking, a detrimental trait in tomato production ${ }^{17}$. In the FRUIT WEIGHT 2.2 (FW2.2) locus, we identified two independent homozygous deletions that disrupted the coding frame of exon 2 (Supplementary Fig. 3). Surprisingly, despite these mutations, we did not detect any discernible change in fruit sizes in these $\mathrm{T}_{2}$ mutant lines compared with WT S. pimpinellifolium (Supplementary Table 4).

During the course of our experimental program, we learned that the gene locus responsible for the multilocular fruit phenotype of FAS is not encoded by a YABBY transcription factor, as was previously proposed $^{18}$, but instead results from a mutation in CLAVATA3 $(C L V 3)^{12}$. Therefore, we carried out a second round of genome engineering to generate multiplex-edited plants in which the selection of target genes focused on modulating fruit size (FW2.2, FAS) and number (MULT), as well as nutritional value ( $L Y C O P E N E$ BETA CYCLASE, CycB). We designed a CRISPR-Cas9 plant transformation vector named pTC603 (Supplementary Note 2), which harbored eight gRNAs targeting two sites in the coding region of each of these four genes (Supplementary Fig. 1). Three successfully transformed $T_{0}$ lines were identified (Supplementary Fig. 4). Sequencing of $\mathrm{T}_{0}$ plants 5 and 8 identified loss-of-function mutations in all four targeted loci in both lines (Supplementary Fig. 5 and Supplementary Dataset 2). Target locus analysis in 28 individual $\mathrm{T}_{1}$ representatives derived from both $\mathrm{T}_{0}$ plants 5 and 8 revealed that in every $\mathrm{T}_{1}$ plant all four target loci were successfully edited (Supplementary Dataset 1). In $C y c B$ and FW2.2 we identified two different mutation events and in CLV3 three different mutation events homozygous mutations or heterozygous (biallelic) combinations of mutant alleles. Either type of mutation resulted in a loss of function of the edited gene. For MULT, we recovered $5 \mathrm{~T}_{1}$ plants possessing two
WT alleles and 23 heterozygous $\mathrm{T}_{1}$ plants (Supplementary Table 5). We examined the two most closely related genomic sequences for each gRNA (as determined by in silico analysis using the Geneious R11 program) and did not observe off-target editing in ten analyzed $\mathrm{T}_{1}$ plants (Supplementary Fig. 2). To summarize, in two separate experiments we engineered two sets of four target domestication genes simultaneously and generated a diverse range of combinations of 15 independent loss-of-function alleles (Supplementary Tables 3 and 4).

We phenotyped the second set of edited plant lines. Introduction of a loss-of-function MULT allele into cultivated tomato resulted in a higher number of fruits per truss, conferring enhanced yield ${ }^{14}$. In our edited plant lines, we identified a single heterozygous mutation (2-bp deletion) in exon 2 (Fig. 2a and Supplementary Fig. 5) of MULT. Edited plants had the predicted branched inflorescence phenotype $\left(T_{1}\right.$, Fig. 2b-d; $\mathrm{T}_{0}$, Supplementary Fig. 6). Both $\mathrm{T}_{1}$ lines also harbored homozygous indel mutations in both the first and the second exon of the FW2.2 gene (Supplementary Fig. 5). Moreover, $\mathrm{T}_{1}$ lines 5 and 8 also carried biallelic deletions in exon 1 and a heterozygous deletion in exon 2 of the CLV3 gene (Fig. 2e), which corresponded with phenotypes that have been attributed to the FAS locus (Fig. 2f,g). Characterization of fruit morphology revealed a fourfold increase in fruit locule number and a fruit weight increased up to $200 \%$ compared with S. pimpinellifolium (Fig. 2h,i). Considering the absence of a discernible fruit phenotype in the lines in which FW2.2 but not FAS was mutated (Supplementary Fig. 3 and Supplementary Table 4), we conclude that increased fruit size (Fig. 2j,k) is due to the loss of function of CLV3. Since the classical $f w 2.2$ mutation, which affects fruit size, causes a heterochronic alteration in the FW2.2 expression pattern, we suggest that changes in FW2.2 expression, but not loss of function, affect tomato fruit size ${ }^{13}$.

Nutritional value and taste have largely been neglected in conventional breeding ${ }^{19}$. The content of lycopene and $\beta$-carotene largely determines the nutritional value of tomato ${ }^{20}$. Anti-inflammatory properties and the reduction of cardiovascular and cancer risks have also been correlated with dietary intake of lycopene ${ }^{21}$. In addition, while $\beta$-carotene occurs in sufficient amounts in many vegetables, lycopene is generally not present in sufficient concentration ${ }^{22}$ In cultivated tomato, the activity of lycopene $\beta$-cyclase converts a substantial fraction of lycopene into $\beta$-carotene, thereby reducing the content of beneficial lycopene ${ }^{15}$. While cultivated cherry tomato fruits accumulate around $60-120 \mathrm{mg} \mathrm{kg}^{-1}$ lycopene $\mathrm{e}^{23,24}$, this antioxidant accumulates to levels of up to $270 \mathrm{mg} \mathrm{kg}^{-1}$ in S. pimpinellifolium ${ }^{25,26}$. The genetic bases determining lycopene accumulation in tomato are only poorly understood and most likely polygenic $^{27}$. Accordingly, previous attempts to enhance lycopene 
content of commercial tomato yielded only moderate success or resulted in enhanced lycopene accumulation at the cost of reduced $\beta$-carotene abundance $e^{15,28}$.
We targeted the $C y c B$ gene in both experiments to evaluate whether we could exploit the lycopene accumulation phenotype of S. pimpinellifolium. In all four edited S. pimpinellifolium lines, we detected biallelic

a

a
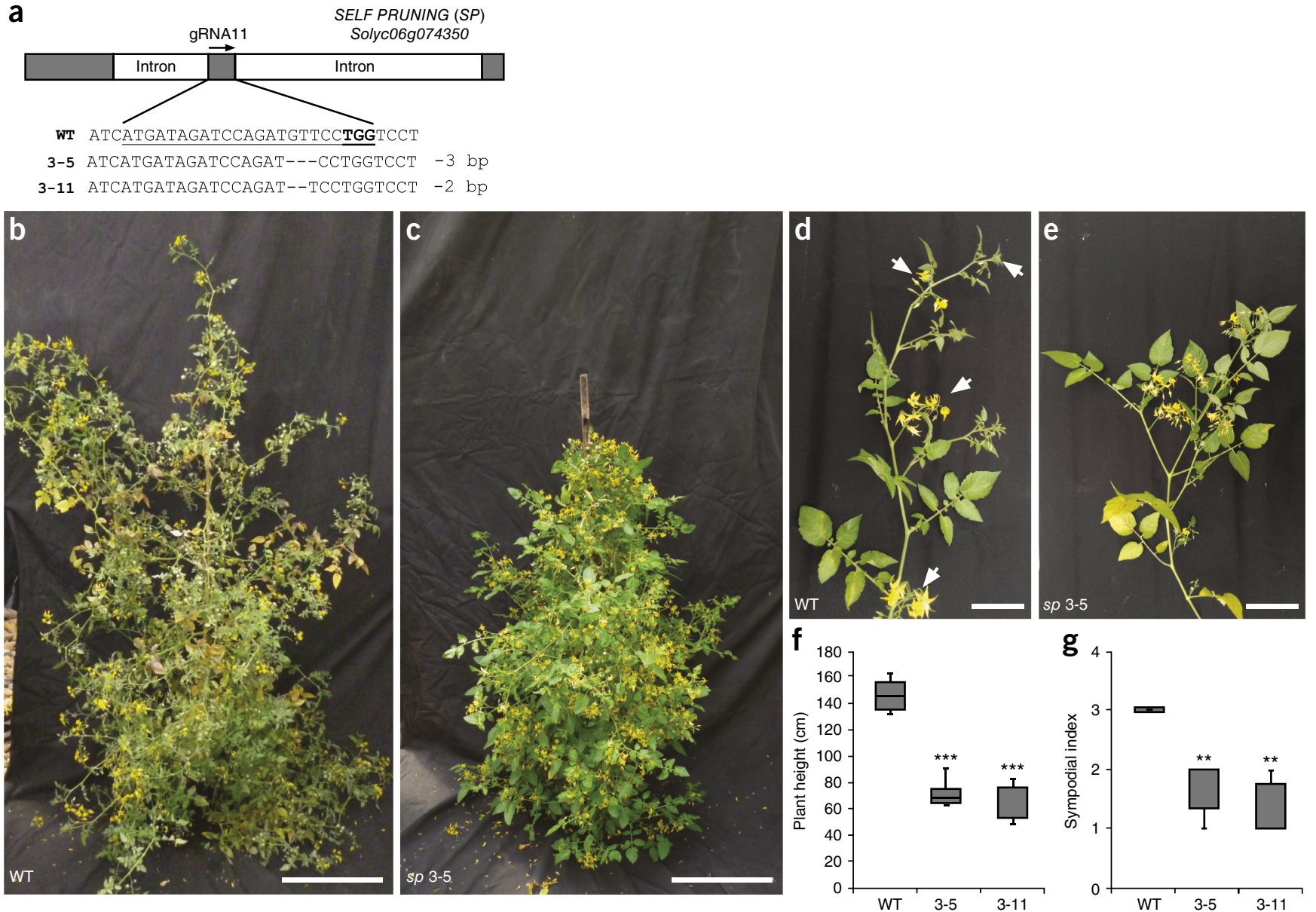

h

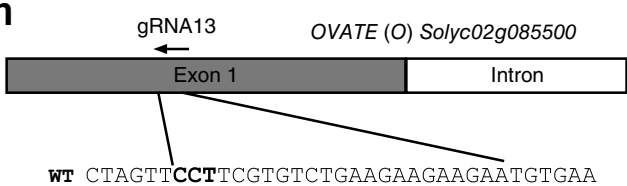

3-5 CTAGTTCCTTCGTG---AAGAAGAAGAATGTGAA -4 bp

3-11 CTAGTTCCTTCGT--CTGAAGAAGAAGAATGTGAA -2 bp
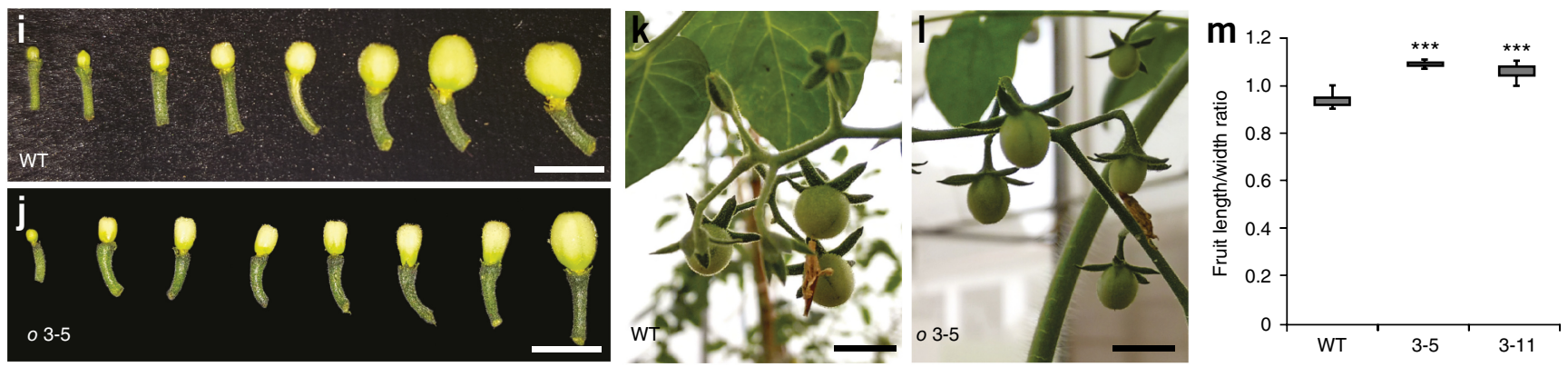

Figure 1 Plant morphology and fruit shape in de novo domesticated S. pimpinellifolium plants. (a) Genomic sequence showing the gRNA target site (underlined) and the resulting missense mutation in the SP gene. The PAM sequence is depicted in bold. (b,c) Representative WT (b) and $T_{2} s p$ mutant (c) plants with determinate growth habit; bars, $30 \mathrm{~cm}$. (d,e) Detailed view of the vegetative branch with successive sympodial units (inflorescence plus three leaves; white arrows) in WT (d) and $\mathrm{T}_{2} s p$ mutant $(\mathbf{e})$; bars, $5 \mathrm{~cm}$. (f,g) Height (f) and sympodial index (g) alterations in WT and $s p$ mutant plants. (h) Genomic sequence showing the site of gRNA targeting and the resulting missense mutation in the $O$ gene. (i-I) Developmental series of early fruits of WT (i) and $\mathrm{T}_{2}$ ovate $(0)$ mutant (j) plants (bars, $1 \mathrm{~cm}$ ) and their respective unripe fruits in the plant $(\mathbf{k}, \mathbf{l}) ;$ bars, $5 \mathrm{~cm}$. ( $\mathrm{m}$ ) Fruit length/width ratio in WT and line 3-5 and 3-11 mutant plants. Two-tailed $t$-test (WT vs. lines): ${ }^{*} P<0.01$ and ${ }^{* * *} P<0.001 ; n=6$ plants for height and sympodial index and $n=90$ fruits for fruit length/width ratio. Data are depicted in box plots: box, interquartile range (IQR); whiskers, $1.5 \times I Q R ;$ center line, mean. 
or homozygous loss-of-function mutations in the first exon of $C y c B$ (Fig. 3a and Supplementary Figs. 5 and 7). These genetic alterations resulted in flowers with orange antheridial cones (due to lycopene accumulation) rather than the yellow anthers of $S$. pimpinellifolium lines 5 and 8 (Fig. $\mathbf{3 b}$-d) and resulted in deep red fruits (Fig. $\mathbf{3 e}, \mathbf{f}$ ). Quantitative determination of the carotenoid content in fruits of cherry

a
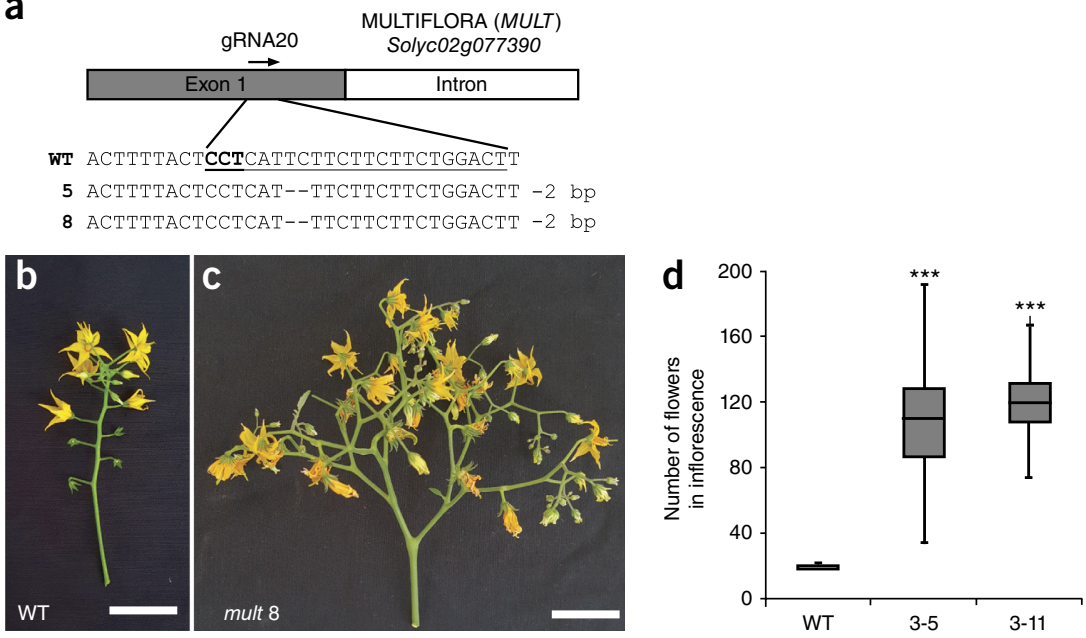

e

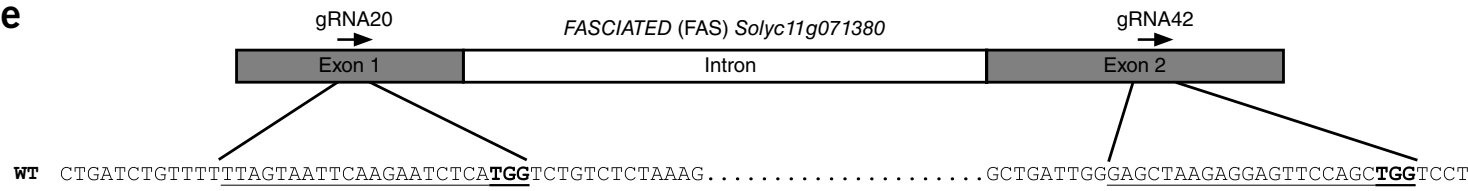

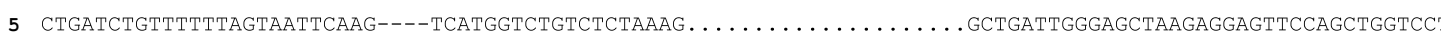
СTGATCTGTTTTTTAGTAATTCAAGAAT-TCATGGTCTGTCTCTAAAG . . . . . . . . . . . . . . . . . . . . . CTGATCTGTTTTTTAGTAATTCAAG----TCATGGTCTGTCTCTAAAG . . . . . . . . . . . . . CTGATCTGTTTTTTAGTAATTCAAGAAT-TCATGGTCTGTCTCTAAAG . . . . . . . . . . .

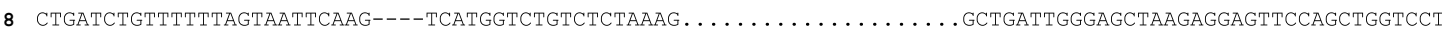

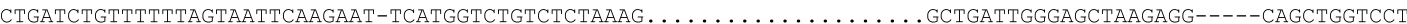
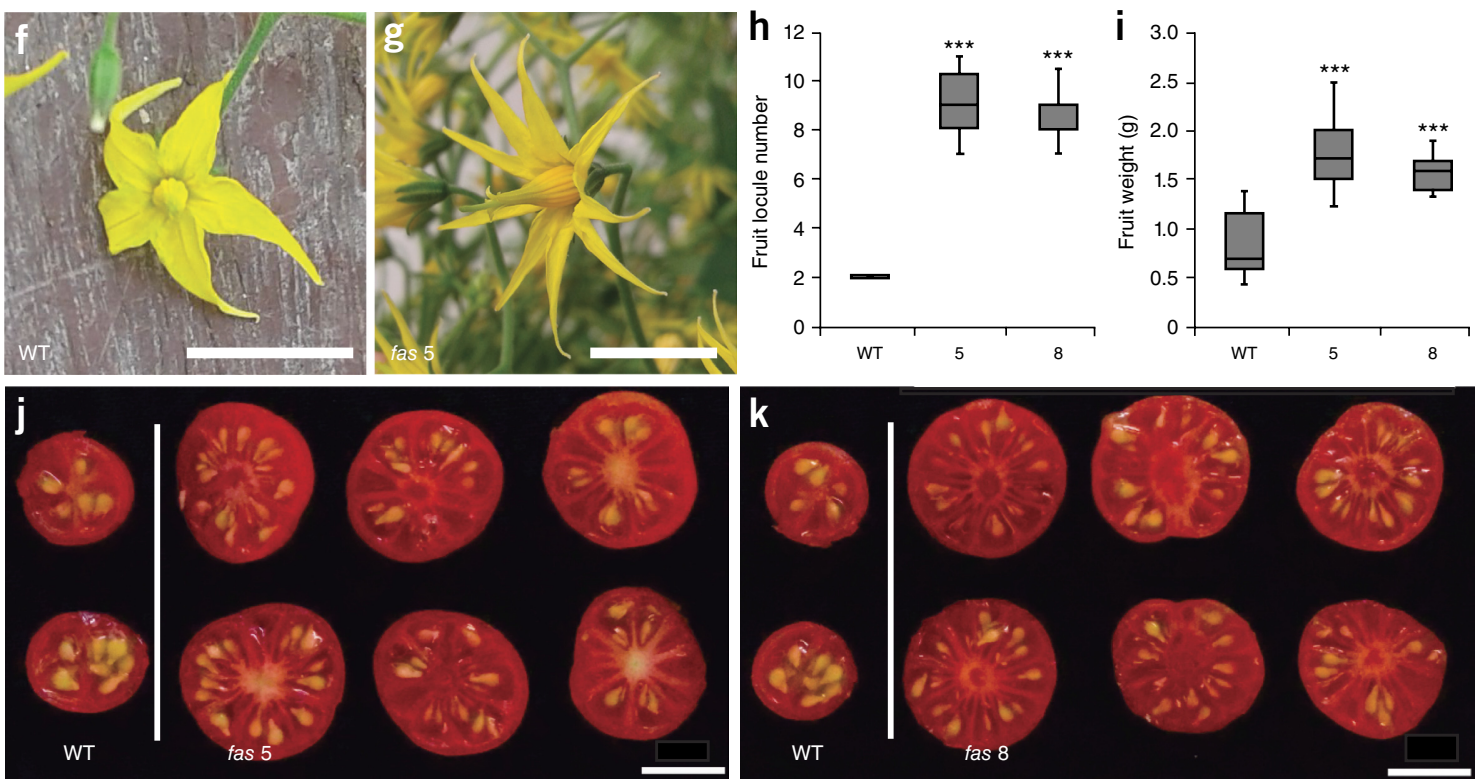

Figure 2 Flower number and fruit size in de novo domesticated S. pimpinellifolium plants. (a) MULT genomic sequence showing the site of gRNA targeting and the resulting missense mutations. (b,c) Representative WT (b) and $\mathrm{T}_{1}$ multiflora (mult) (c) mutant inflorescences. Note the increased number of petals and their intense yellow color due to the mutated fas and $c y c B$ alleles targeted in the same vector. Bars, $2 \mathrm{~cm}$. (d) Number of flowers per inflorescence in WT and two different $T_{1}$ mutants. (e) clv3 genomic sequence (of the fas allele) showing the site of gRNA targeting and the resulting missense mutations. (f,g) Macroscopic flower morphology in WT (f) and $\mathrm{T}_{1}$ multi-petal fas (g) mutant plants; bars, $2 \mathrm{~cm}$. (h,i) Fruit locules (h) and weight (i) in WT and two different mutant plant lines (derived from events 5 and 8). Increased locule number led to increased fruit size in both fas line 5 T 1 (j) and fas line $8 T_{1}(\mathbf{k})$ mutant plant lines. Scale bars, $1 \mathrm{~cm}$. Two-tailed $t$-test (WT vs. lines): ${ }^{* * *} P<0.001 ; n=60$ inflorescences for flower number per inflorescence and $n=90$ fruits for fruit locule number and weight. Data are depicted in box plots: box, IQR; whiskers, $1.5 \times$ IQR; center line, mean. 
a

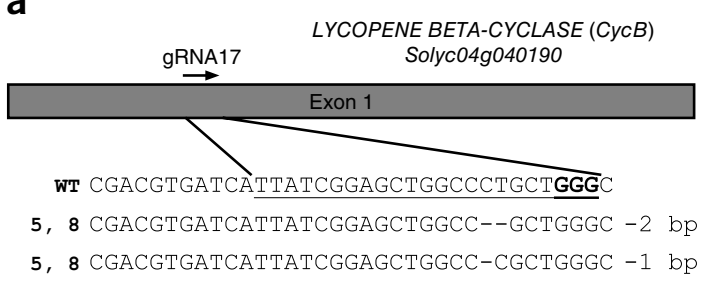

b

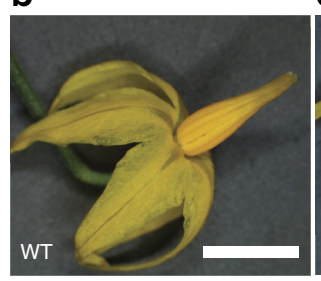

C

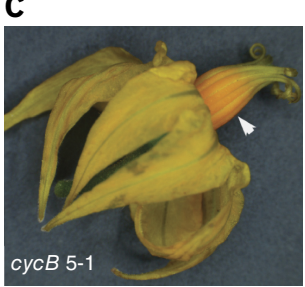

d

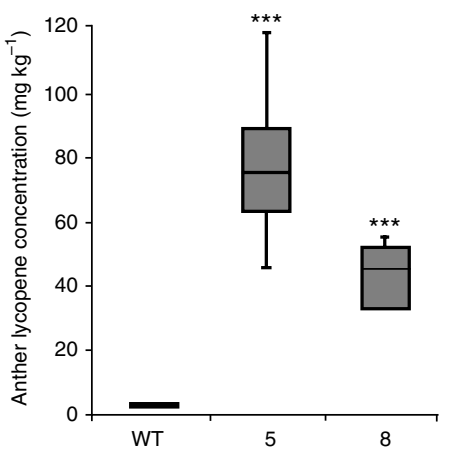

e

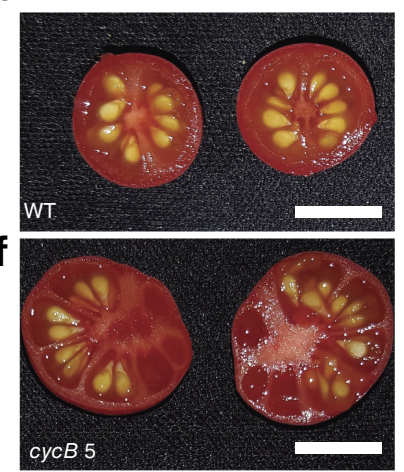

g

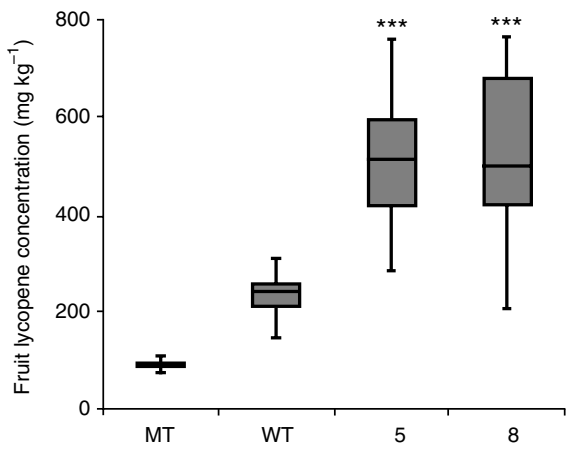

Figure 3 Nutritional content of fruits from engineered S. pimpinellifolium plants. (a) CycB genomic sequence showing the site of gRNA targeting and the resulting missense mutations in plants 5 and 8 created using vector pTC603. (b,c) Close-up of (b) WT and (c) cyc $B \mathrm{~T}_{1}$ mutant flowers; arrowhead shows anther color alteration due to lycopene accumulation; bar, $2 \mathrm{~cm}$ in b,c. (d) Lycopene concentration in the antheridial cones of WT and two different $\mathrm{T}_{1}$ mutant plant lines (derived from events 5 and 8 ). (e,f) Cross-sections of representative $T_{1}$ fruits showing intensification of the red color in $c y c B$ mutant plants; bar, $1 \mathrm{~cm}$. (g) Lycopene concentration in mature fruits of cherry tomato cultivar Micro-Tom (MT), S. pimpinellifolium (WT) and two different S. pimpinellifolium mutant lines (derived from events 5 and 8 ). One-way ANOVA followed by Tukey's HSD test (mutants vs. WT): *** $P<0.001 . n=6$ plants (pooled flowers or fruits) for lycopene content in flowers or fruits. Data are depicted in box plots: box, IQR; whiskers, $1.5 \times$ IQR; center line, mean.

tomatoes cv. Micro-Tom (MT) and those of wild and edited S. pimpinellifolium lines revealed values of 92, 234, and $510 \mathrm{mg} \mathrm{kg}^{-1}$ of lycopene, respectively (Fig. 3g and Supplementary Tables 6 and 7). This represents more than $100 \%$ higher accumulation of lycopene in the edited lines when compared with the parent species. Further, this value is more than $500 \%$ higher than concentrations commonly found in commercial cherry tomatoes ${ }^{23,24}$. Importantly, this improvement in lycopene content did not negatively affect the accumulation of $\beta$-carotene or lutein (Supplementary Fig. 8).

Finally, we monitored the stability of the observed phenotypes in the $\mathrm{T}_{2}$ (pTC603) and $\mathrm{T}_{3}$ (pTC321) generations and verified their occurrence independent of the Cas 9 transgene. Diagnostic PCR analyses identified transgene-free plants that had all of the domestication phenotypes (Supplementary Fig. 9). Important traits such as Brix value, fruit shape and locule number were uniformly inherited in the $\mathrm{T}_{2}$ (pTC603) and $\mathrm{T}_{3}$ (pTC321) generations (Supplementary Figs. 10 and 11 and Supplementary Tables 8 and 9 )

In this report we provide evidence that targeted reverse genetic engineering of wild plants could rapidly create new crops. We demonstrate that simultaneous CRISPR-Cas9 editing of six genes resulted in modification of fruit number, size, shape, nutrient content and plant architecture in a single generation and within a single transformation experiment. Recently, a complementary promoter editing approach has been applied to create genetic variability and reverse the negative effects of domestication history in order to aid breeding of beneficial traits in tomato $^{29}$. Combining this synthetic genetic variability method with de novo domestication could boost efforts to rapidly engineer better crops. Importantly, domestication traits have also been characterized in many crops, including maize, wheat and sorghum ${ }^{6,30}$, meaning that our approach could be broadly applied ${ }^{9}$.

\section{METHODS}

Methods, including statements of data availability and any associated accession codes and references, are available in the online version of the paper.

Note: Any Supplementary Information and Source Data files are available in the online version of the paper.

\section{ACKNOWLEDGMENTS}

We are grateful to S. Schültke for technical assistance. This work was supported by funding from the Agency for the Support and Evaluation of Graduate Education (CAPES, Brazil), the National Council for Scientific and Technological Development (CNPq, Brazil) and Foundation for Research Assistance of the São Paulo State (FAPESP, Brazil), and the German Federal Ministry of Education and Research (BMBF, Germany). We thank CAPES for studentships granted to E.R.N. and FAPESP for the studentship granted to M.M.N. (2013/12209-1). L.F. was supported by FAPESP grant 2013/18056-2. FAPESP and BMBF provided a grant for L.E.P.P. (2015/50220-2) and J.K. (031B0334). L.E.P.P. acknowledges a grant from CNPq (grant 307040/2014-3).

\section{AUTHOR CONTRIBUTIONS}

A.Z., T.C., D.F.V., J.K. and L.E.P.P. designed the study. A.Z., T.C., M.M.N., E.R.N., K.H.E., S.W. and L.F. performed experiments. A.Z., T.C. and K.H.E. analyzed data. A.Z., K.H.E., J.K. and L.E.P.P. prepared the manuscript. All authors have revised and approved the final version of the manuscript.

\section{COMPETING INTERESTS}

After completion of this work in the laboratory of D.F.V., T.C. became an employee of Inari Agriculture, a company that uses novel technologies for crop breeding. D.F.V. is a founder and Chief Science Officer of Calyxt, a company applying genome editing to plants.

Reprints and permissions information is available online at http://www.nature.com/ reprints/index.html. Publisher's note: Springer Nature remains neutral with regard to jurisdictional claims in published maps and institutional affiliations.

1. Evans, L.T. Crop Evolution, Adaptation and Yield (Cambridge Univ. Press, 1996). 
2. van de Wouw, M. Kik, C, van Hintum, T, van Treuren, R \& Visser, B. Genetic erosion in crops: concept, research results and challenges. Plant Genet. Resour. 8, $1-15$ (2010).

3. Food and Agriculture Organization of the United Nations. FAO Statistical Yearbook 2015: World Food and Agriculture (United Nations, 2015).

4. Lippman, Z. \& Tanksley, S.D. Dissecting the genetic pathway to extreme fruit size in tomato using a cross between the small-fruited wild species Lycopersicon pimpinellifolium and L. esculentum var. Giant Heirloom. Genetics 158, 413-422 (2001).

5. Gruber, K. Agrobiodiversity: the living library. Nature 544, S8-S10 (2017).

6. Meyer, R.S. \& Purugganan, M.D. Evolution of crop species: genetics of domestication and diversification. Nat. Rev. Genet. 14, 840-852 (2013).

7. Cermák, T. et al. A multipurpose toolkit to enable advanced genome engineering in plants. Plant Cell 29, 1196-1217 (2017).

8. Falke, K.C. et al. The spectrum of mutations controlling complex traits and the genetics of fitness in plants. Curr. Opin. Genet. Dev. 23, 665-671 (2013).

9. Zsögön, A., Cermak, T., Voytas, D. \& Peres, L.E.P. Genome editing as a tool to achieve the crop ideotype and de novo domestication of wild relatives: case study in tomato. Plant Sci. 256, 120-130 (2017).

10. Pnueli, L. et al. The SELF-PRUNING gene of tomato regulates vegetative to reproductive switching of sympodial meristems and is the ortholog of CEN and TFL1. Development 125, 1979-1989 (1998).

11. Liu, J., Van Eck, J., Cong, B. \& Tanksley, S.D. A new class of regulatory genes underlying the cause of pear-shaped tomato fruit. Proc. Natl. Acad. Sci. USA 99, 13302-13306 (2002)

12. Xu, C. et al. A cascade of arabinosyltransferases controls shoot meristem size in tomato. Nat. Genet. 47, 784-792 (2015)

13. Frary, A. et al. Fw2.2: a quantitative trait locus key to the evolution of tomato fruit size. Science 289, 85-88 (2000).

14. Lippman, Z.B. et al. The making of a compound inflorescence in tomato and related nightshades. PLoS Biol. 6, e288 (2008).

15. Ronen, G., Carmel-Goren, L., Zamir, D. \& Hirschberg, J. An alternative pathway to $\beta$-carotene formation in plant chromoplasts discovered by map-based cloning of beta and old-gold color mutations in tomato. Proc. Natl. Acad. Sci. USA 97, 11102-11107 (2000)

16. Rick, C.M. The tomato. Sci. Am. 239, 76-87 (1978).

17. Peet, M.M. Fruit cracking in tomato. Horttechnology 2, 216-223 (1991).

18. Cong, B., Barrero, L.S. \& Tanksley, S.D. Regulatory change in YABBY-like transcription factor led to evolution of extreme fruit size during tomato domestication. Nat. Genet. 40, 800-804 (2008).

19. Tieman, D. et al. A chemical genetic roadmap to improved tomato flavor. Science 355, 391-394 (2017).

20. Römer, S. et al. Elevation of the provitamin A content of transgenic tomato plants. Nat. Biotechnol. 18, 666-669 (2000).

21. Clinton, S.K. Lycopene: chemistry, biology, and implications for human health and disease. Nutr. Rev. 56, 35-51 (2009).
22. Bramley, P.M. Is lycopene beneficial to human health? Phytochemistry 54, 233-236 (2000).

23. Lenucci, M.S., Cadinu, D., Taurino, M., Piro, G. \& Dalessandro, G. Antioxidant composition in cherry and high-pigment tomato cultivars. J. Agric. Food Chem. 54 2606-2613 (2006)

24. Kuti, J.O. \& Konuru, H.B. Effects of genotype and cultivation environment on lycopene content in red-ripe tomatoes. J. Sci. Food Agric. 85, 2021-2026 (2005).

25. Adalid, A.M., Roselló, S. \& Nuez, F. Evaluation and selection of tomato accessions (Solanum section Lycopersicon) for content of lycopene, $\beta$-carotene and ascorbic acid. J. Food Compos. Anal. 23, 613-618 (2010).

26. Ashrafi, H., Kinkade, M.P., Merk, H.L. \& Foolad, M.R. Identification of nove quantitative trait loci for increased lycopene content and other fruit quality traits in a tomato recombinant inbred line population. Mol. Breed.30, 549-567 (2012).

27. Liu, Y.-S. There is more to tomato fruit colour than candidate carotenoid genes. Plant Biotechnol. J. 1, 195-207 (2003).

28. Li, C. et al. RNA-guided Cas9 as an in vivo desired-target mutator in maize. Plant Biotechnol. J. 15, 1566-1576 (2017).

29. Soyk, S. et al. Bypassing negative epistasis on yield in tomato imposed by a domestication gene. Cell 169, 1142-1155.e12 (2017).

30. Doebley, J.F., Gaut, B.S. \& Smith, B.D. The molecular genetics of crop domestication. Cell 127, 1309-1321 (2006).

31. Doebley, J., Stec, A. \& Hubbard, L. The evolution of apical dominance in maize Nature 386, 485-488 (1997)

32. Studer, A., Zhao, Q., Ross-Ibarra, J. \& Doebley, J. Identification of a functional transposon insertion in the maize domestication gene tb1. Nat. Genet. 43, 1160-1163 (2011).

33. Moreno, M.A., Harper, L.C., Krueger, R.W., Dellaporta, S.L. \& Freeling, M. liguleless encodes a nuclear-localized protein required for induction of ligules and auricles during maize leaf organogenesis. Genes Dev. 11, 616-628 (1997).

34. Wang, H., Studer, A.J., Zhao, Q., Meeley, R. \& Doebley, J.F. Evidence that the origin of naked kernels during maize domestication was caused by a single amino acid substitution in tga1. Genetics 200, 965-974 (2015).

35. Yang, Q. et al. CACTA-like transposable element in ZmCCT attenuated photoperiod sensitivity and accelerated the postdomestication spread of maize. Proc. Natl. Acad. Sci. USA 110, 16969-16974 (2013).

36. Huang, C. et al. ZmCCT9 enhances maize adaptation to higher latitudes. Proc Natl. Acad. Sci. USA 115, E334-E341 (2018).

37. Tian, Z. et al. Artificial selection for determinate growth habit in soybean. Proc Natl. Acad. Sci. USA 107, 8563-8568 (2010)

38. Cai, Y. et al. CRISPR/Cas9-mediated targeted mutagenesis of GmFT2a delays flowering time in soya bean. Plant Biotechnol. J. 16, 176-185 (2018).

39. Lu, X. et al. The transcriptomic signature of developing soybean seeds reveals the genetic basis of seed trait adaptation during domestication. Plant J. 86, 530-544 (2016).

40. Dong, Y. et al. Pod shattering resistance associated with domestication is mediated by a NAC gene in soybean. Nature Commun. 5, 3352 (2014). 


\section{ONLINE METHODS}

Molecular cloning and plant transformation. CRISPR-Cas9 plant transformation vectors were constructed using protocols $3 \mathrm{~A}$ and 5 of Čermák et al. ${ }^{7}$. The vector pTC321 (35S::Csy4-P2A-AtCas9, 35S::gRNA-array) expressing a Csy4 array of six gRNAs targeting SELF PRUNING (Solyc06g074350), OVATE (Solyc02g085500), FRUIT WEIGHT 2.2 (Solyc02g090730), LYCOPENE BETA-CYCLASE (Solyc04g040190), MULTIFLORA (Solyc02g077390) and FASCIATED/YABBY (Solyc11g071810) was constructed by direct assembly of gRNAs into the T-DNA vector pDIRECT_22C (Addgene plasmid 91135) (see Supplementary Table 10 for gRNA sequences). Vector pTC603 (35S:: Csy4-P2A-AtCas9, CmYLCV::gRNA-array) expressing a Csy4 array of eight gRNAs targeting FASCIATED/CLAVATA3 (Solyc11g071380), FRUIT WEIGHT 2.2 (Solyc02g090730), MULTIFLORA (Solyc02g077390) and LYCOPENE BETA-CYCLASE (Solyc04g040190) was constructed in two steps. The first six gRNAs were cloned into pMOD_B2103 (Addgene plasmid 91061) and the last two gRNAs into pMOD_C2200 (Addgene plasmid 91082). The gRNA arrays in the resulting plasmids were assembled along with pMOD_A0501 (35S:Csy4-P2A-AtCas9, Addgene plasmid 91011) into the T-DNA vector pTRANS_220 (Addgene plasmid 91113). The final array contains the gRNAs in the following order: gRNAs 41 and 42 targeting FAS/CLV3, gRNAs 15 and 16 targeting FW2.2, gRNAs 19 and 20 targeting MULTIFLORA and gRNAs 17 and 18 targeting $C y c B$ (Supplementary Fig. 1 and Supplementary Table 10).

The pTC321 (Supplementary Note 1) and pTC603 (Supplementary Note 2) vectors were introduced into the Agrobacterium tumefaciens strain LBA4404 by electroporation. Agrobacteria were cultured in $3 \mathrm{~mL}$ of liquid LB medium supplemented with $50 \mathrm{mg} \mathrm{L}^{-1}$ kanamycin and $50 \mathrm{mg} \mathrm{L}^{-1}$ rifampicin and incubated at $28{ }^{\circ} \mathrm{C}$ for $24 \mathrm{~h}$ at 120 r.p.m. From this culture, $500 \mu \mathrm{L}$ were added to $50 \mathrm{~mL}$ of fresh LB medium with the same antibiotics as above. The culture was incubated overnight under the same conditions and then centrifuged at $2,000 \mathrm{~g}$ for $15 \mathrm{~min}$ at room temperature. The pellet was resuspended in liquid Murashige and Skoog (MS) medium ${ }^{41}$ to an $\mathrm{OD}_{600}$ of $0.25-0.3$; acetosyringone was added to a final concentration of $100 \mu \mathrm{M}$ before plant inoculation.

S. pimpinellifolium (LA1589) was transformed as previously published ${ }^{42}$ with the following modifications: seeds were surface-sterilized by shaking in 20 $\mathrm{mL} 30 \%(\mathrm{v} / \mathrm{v})$ commercial bleach (2.7\% sodium hypochlorite) supplemented with two drops of commercial detergent for $15 \mathrm{~min}$, followed by three rinses with sterile water. Seeds were germinated on semi-solid MS medium (supplemented with $0.6 \mathrm{~g} / \mathrm{L}$ agar) and incubated at $25 \pm 1{ }^{\circ} \mathrm{C}$ in the dark for $4 \mathrm{~d}$. After this period, seeds were transferred and maintained at $25 \pm 1{ }^{\circ} \mathrm{C}$ under long-day conditions ( $16 \mathrm{~h}$ light/ $8 \mathrm{~h}$ dark) with $45 \mu \mathrm{mol}$ photons $\mathrm{m}^{-2} \mathrm{~s}^{-1}$ PAR irradiance.

Leaf explants $\left(1-2 \mathrm{~cm}^{2}\right)$ were isolated from 2 -week-old in vitro plants and placed with the abaxial side down onto MS medium containing $1 \mathrm{mg} \mathrm{L}^{-1}$ trans-zeatin, $0.1 \mathrm{mg} \mathrm{L}^{-1}$ indole-3-acetic acid (IAA) and $100 \mu \mathrm{M}$ acetosyringone. Two drops of Agrobacterium suspension were applied per explant and plates were then incubated at room temperature for $10 \mathrm{~min}$. Thereafter, excess bacterial suspension was removed, and explants were blotted dry on sterile filter paper. Plates were maintained in the dark at $25^{\circ} \mathrm{C}$ for $2 \mathrm{~d}$ for cocultivation. Explants were transferred to plates with MS medium containing $1 \mathrm{mg} \mathrm{L}^{-1}$ trans-zeatin, $0.1 \mathrm{mg} \mathrm{L}^{-1} \mathrm{IAA}, 300 \mathrm{mg} \mathrm{L}^{-1}$ timentin, $100 \mathrm{mg} \mathrm{L}^{-1}$ kanamycin. After 5 weeks, shoot primordia emerging from callus tissue were isolated and transferred to shoot elongation medium (MS medium supplemented with $0.5 \mathrm{mg} \mathrm{L}^{-1}$ trans-zeatin, $300 \mathrm{mg} \mathrm{L}^{-1}$ timentin, $100 \mathrm{mg} \mathrm{L}^{-1}$ kanamycin) to recover full plants. After acclimation, these plants were grown in a greenhouse at $30^{\circ} \mathrm{C} / 26^{\circ} \mathrm{C}$ day/night temperature and $60-75 \%$ ambient relative humidity, $11.5 \mathrm{~h} / 13 \mathrm{~h}$ (winter/summer) photoperiod, sunlight 250-350 $\mu \mathrm{mol}$ photons $\mathrm{m}^{-2} \mathrm{~s}^{-1}$ PAR irradiance, attained by a reflecting mesh (Aluminet, Polysack Indústrias Ltda, Leme, Piracicaba, SP, Brazil), and automatic irrigation four times a day for fruit set.

Seeds from transformed plants were germinated in $350-\mathrm{mL}$ pots with a 1:1 mixture of commercial potting mix Basaplant (Base Agro, Artur Nogueira, SP, Brazil) and expanded vermiculite supplemented with $1 \mathrm{~g} \mathrm{~L}^{-1}$ 10:10:10 NPK and $4 \mathrm{~g} \mathrm{~L}^{-1}$ dolomite limestone $\left(\mathrm{MgCO}_{3}\right.$ plus $\left.\mathrm{CaCO}_{3}\right)$. Upon appearance of the first true leaf, seedlings of each genotype were transplanted to $10-\mathrm{L}$ pots containing the soil mix described above, except for NPK supplementation, which was increased to $8 \mathrm{~g} \mathrm{~L}^{-1}$. After transplanting, plants were sprayed twice at $14-\mathrm{d}$ intervals with $1 \mathrm{~g} \mathrm{~L}^{-1}$ Peters 20-20-20 leaf fertilizer. Acclimatized plants were pollinated by hand, producing $\mathrm{T}_{1}$ seeds and the following generations.

Seeds and vectors are available upon request from the corresponding authors see (https://www.uni-muenster.de/Biologie.IBBP/agkudla/Plasmids.html).

Genotyping of $T_{0}$ and $T_{1}$ plants. The gRNA target sites were amplified by PCR directly from leaf tissue with the Phire Plant Direct PCR Master Mix following the Dilution \& Storage protocol (Thermo Fisher Scientific) or from extracted genomic DNA with Phusion polymerase (New England BioLabs), using primers listed in Supplementary Table 11. Primers and dNTPs were removed using ExoSAP ( $0.5 \mathrm{U}$ exonuclease $\mathrm{I}+0.25 \mathrm{U}$ FastAP thermosensitive alkaline phosphatase in $35 \mu \mathrm{L}$ of PCR, incubated $30 \mathrm{~min}$ at $37^{\circ} \mathrm{C}$ and heatinactivated for $5 \mathrm{~min}$ at $95^{\circ} \mathrm{C}$ ) and the purified PCR products were directly sequenced. Heterozygous and biallelic mutations were identified as overlapping sequence traces. These samples were cloned and several clones sequenced to determine the sequences of individual alleles.

Off-target identification and analysis. The "Find CRISPR sites" function in Geneious R11 was used to search for off-target binding sites in S. pimpinellifolium genome ${ }^{43}$ (https://solgenomics.net/organism/Solanum_pimpinellifolium/genome). A maximum of eight mismatches and zero indels were allowed between the on- and off-target sequences. The two top scoring off-target sites were PCR amplified and sequenced. Primers can be found in Supplementary Table 12.

Plant material and growth conditions. Plants were cultivated in semicontrolled conditions in a greenhouse in Viçosa (642 $\mathrm{m}$ asl, $\left.20^{\circ} 45^{\prime} \mathrm{S}, 42^{\circ} 51^{\prime} \mathrm{W}\right)$, Minas Gerais, Brazil. Wild-type S. pimpinellifolium plants were grown alongside $\mathrm{T}_{2}$ of pTC321-, $\mathrm{T}_{3}$ of pTC321- and $\mathrm{T}_{1}$ of pTC603-transformed plants during the months of October 2016 to January 2017, August 2017 to November 2017 and August 2017 to November 2017, respectively. For plants of generations $\mathrm{T}_{3}$ of $\mathrm{pTC} 321$ and $\mathrm{T}_{1}$ of $\mathrm{pTC} 603$, the main stem was trained on bamboo sticks and side branches were pruned, leaving a total of three inflorescences per plant. The greenhouse temperature ranged between 24 and $20{ }^{\circ} \mathrm{C}$, with a 13-h/11-h (day/night) photoperiod and an average irradiance of $800 \mu \mathrm{mol}$ photons $\mathrm{m}^{-2} \mathrm{~s}^{-1}$. Seeds were germinated in plastic trays with commercial substrate Tropstrato and supplemented with $1 \mathrm{~g} \mathrm{~L}^{-1}$ 10:10:10 NPK and $4 \mathrm{~g} \mathrm{~L}^{-1}$ dolomite limestone $\left(\mathrm{MgCO}_{3}\right.$ plus $\left.\mathrm{CaCO}_{3}\right)$. Weekly foliar fertilization was carried out using $2 \mathrm{~g} / \mathrm{L}$ Biofert leaf fertilizer. Upon appearance of the first true leaves, seedlings of each genotype were transplanted to 5 -L pots. The new pots were filled with substrate as described above, except for the NPK supplementation, which was increased to $8 \mathrm{~g} \mathrm{~L}^{-1}$. Irrigation was performed twice a day in a controlled manner, so that each vessel received the same volume of water.

Carotenoid quantification and sampling. Fruits were harvested at the 'red ripe' stage sequentially over a period of 2 weeks. They were cut in half and the seeds were removed, after which the fruits were immediately frozen in liquid nitrogen and stored in a freezer at $-80^{\circ} \mathrm{C}$ until analysis. Fruit carotenoid extraction was carried out following an adaptation of a previously published protocol $^{44}$. Frozen fruits were ground in a ball mill (Retsch, model MM400) and 200-mg aliquots subsequently collected in 2-mL microtubes. During the extraction, samples were kept cooled on ice in an environment with reduced luminosity (30-50 $\mu \mathrm{mol}$ photons $\mathrm{m}^{-2} \mathrm{~s}^{-1}$ irradiance). $100 \mu \mathrm{L}$ of saturated $\mathrm{NaCl}$ solution $(370 \mathrm{~g} / \mathrm{L})$ were added to each sample and mixed by vortexing for $30 \mathrm{~s}$. $200 \mu \mathrm{L}$ of dichloromethane were added next and mixed by vortexing. Finally, $500 \mu \mathrm{L}$ of hexane:ethyl ether (1:1) were added and the sample homogenized by vortexing. Samples were then centrifuged (Eppendorf 5415R micro-centrifuge) $\left(4^{\circ} \mathrm{C}, 13,000 \mathrm{~g}\right.$ for $5 \mathrm{~min}$ ). The supernatant was then collected and transferred to a $2-\mathrm{mL}$ amber microtube. Addition of hexane:ethyl ether (1:1), vortex agitation, centrifugation and collection of the upper organic phase (supernatant) were repeated three times, until whitening of the samples was observed in the microtube, showing the appropriate extraction of most carotenoids. Samples were completely dried in a vacuum concentrator (CentriVap, Labconco), resuspended in $300 \mu \mathrm{L}$ of ethyl acetate and mixed for $30 \mathrm{~s}$. All supernatant fractions were combined, completely dried by vacuum, filtered through a $0.45 \mu \mathrm{m}$ membrane filter and suspended with $100 \mu \mathrm{L}$ of ethyl acetate. Chromatography was 
carried out on Agilent Technologies series 1100 HPLC system on a normal-phase Phenomenex column (Luna C18; $250 \times 4.6 \mathrm{~mm} ; 5 \mu \mathrm{m}$ particle diameter) with a flow rate of $1 \mathrm{~mL} \mathrm{~min}-1$ and temperature $25^{\circ} \mathrm{C}$. The mobile phase was a gradient of ethyl acetate (A) and acetonitrile:water 9:1 (v/v) (B): 0-4 min: 20\% A; 4-30 min: 20-65\% A; 30-35 min: 65\% A; 35-40 min: $65-20 \%$ A. Eluted compounds were detected between 340 and $700 \mathrm{~nm}$ and quantified at $450 \mathrm{~nm}$. The endogenous metabolite concentration was obtained by comparing the peak areas of the chromatograms with commercial standards (SigmaAldrich).

The extraction of corolla and anther samples were performed in a similar way to that of fruits, but with the following modifications: after harvesting, the samples were lyophilized and stored in a refrigerator until the extraction process. The period between freeze-drying and carotenoid extraction was less than $10 \mathrm{~d}$. Lyophilized samples were ground in reaction tubes with the help of a plastic pestle. Anther samples contained on average $14 \mathrm{mg}$ of dry mass, corolla samples $10 \mathrm{mg}$. Samples were rehydrated in $300 \mu \mathrm{L}$ ultrapure water and subjected to the extraction process and HPLC quantification.

Brix determination. The total content of soluble solids in fruits (measured as Brix) was determined for the 'red ripe' fruits stage by means of a digital bench refractometer (Instrutherm Model RTD-45). First the apparatus was calibrated with distilled water; after drying the prism, the liquid of each fruit was placed in the prism of the refractometer and thus quantified. The Brix of each replicate (each plant) was composed of analyzes of many fruits.

Phenotypic parameters of the fruits. The phenotypic parameters of the fruits (mass, length and equatorial width) were determined using precision balances (Micronal B160) and a digital caliper (MTX) using at least 10 fruits per plant.
Statistics. The quantitative parameters studied here show continuous variation and were therefore analyzed using parametric tests: Student's $t$-test was done on GraphPad; ANOVA and Tukey HSD tests were performed using VassarStats (http://vassarstats.net). Percentage data were converted to the inverse function $(1 / X)$ before analysis. All information on replication, statistical test and presentation are given in the respective figure legends.

Reporting Summary. Further information on research design is available in the Nature Research Reporting Summary linked to this article.

Data availability. The data supporting the findings of this study are available in the paper and the accompanying Supplementary Information files. Specifically, sequences supporting on- and off-target analyses are available in Figures 1-3, Supplementary Figures 2, 3, 5 and 7, and Supplementary Tables 3 and 4 . Raw sequences and alignments for the above mentioned figures and tables are available in Supplementary Datasets 1 and 2. Complete and annotated sequences of pTC321.gb and pTC603.gb are in Supplementary Notes 1 and 2, respectively.

41. Pino, L.E. et al. The Rg1 allele as a valuable tool for genetic transformation of the tomato 'Micro-Tom' model system. Plant Methods 6, 23 (2010).

42. Murashige, T. \& Skoog, F. A revised medium for rapid growth and bio assays with tobacco tissue cultures. Physiol. Plant. 15, 473-497 (1962).

43. Tomato Genome Consortium. The tomato genome sequence provides insights into fleshy fruit evolution. Nature 485, 635-641 (2012).

44. Sérino, S., Gomez, L., Costagliola, G. \& Gautier, H. HPLC assay of tomato carotenoids: validation of a rapid microextraction technique. J. Agric. Food Chem. 57, 8753-8760 (2009). 


\section{Reporting Summary}

Nature Research wishes to improve the reproducibility of the work that we publish. This form provides structure for consistency and transparency in reporting. For further information on Nature Research policies, see Authors \& Referees and the Editorial Policy Checklist.

\section{Statistical parameters}

When statistical analyses are reported, confirm that the following items are present in the relevant location (e.g. figure legend, table legend, main text, or Methods section).

n/a Confirmed

$\bigotimes$ The exact sample size $(n)$ for each experimental group/condition, given as a discrete number and unit of measurement

$\bigotimes$ An indication of whether measurements were taken from distinct samples or whether the same sample was measured repeatedly

$\triangle$ The statistical test(s) used AND whether they are one- or two-sided

Only common tests should be described solely by name; describe more complex techniques in the Methods section.

Х A description of all covariates tested

Х $\square$ A description of any assumptions or corrections, such as tests of normality and adjustment for multiple comparisons A full description of the statistics including central tendency (e.g. means) or other basic estimates (e.g. regression coefficient) AND variation (e.g. standard deviation) or associated estimates of uncertainty (e.g. confidence intervals)

For null hypothesis testing, the test statistic (e.g. $F, t, r$ ) with confidence intervals, effect sizes, degrees of freedom and $P$ value noted Give $P$ values as exact values whenever suitable.

Х $\square$ For Bayesian analysis, information on the choice of priors and Markov chain Monte Carlo settings

Х $\square$ For hierarchical and complex designs, identification of the appropriate level for tests and full reporting of outcomes

Х $\square$ Estimates of effect sizes (e.g. Cohen's $d$, Pearson's $r$ ), indicating how they were calculated

$\varnothing$ Clearly defined error bars

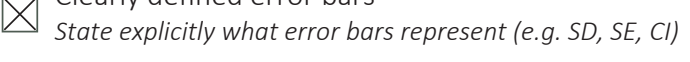

Our web collection on statistics for biologists may be useful.

\section{Software and code}

\section{Policy information about availability of computer code}

Data collection The data collected were basically growth measurements and carotenoid determination, which did need software to be collected.

Data analysis

Statistical analyses were performed using Excel from Windows Office v. 2010 Package. DNA analyses were performed using Geneious v. 9 or DNAStar Lasergene Version 15.

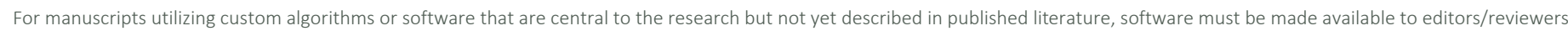

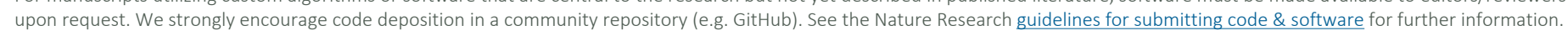

\section{Data}

Policy information about availability of data

All manuscripts must include a data availability statement. This statement should provide the following information, where applicable:

- Accession codes, unique identifiers, or web links for publicly available datasets

- A list of figures that have associated raw data

- A description of any restrictions on data availability

Supplementary Information files. Specifically, sequences supporting on- and off-target analyses are available in Figure 2-4, Supplementary Figure 2, 3, 5 and 7 as 


\section{Field-specific reporting}

Please select the best fit for your research. If you are not sure, read the appropriate sections before making your selection.

$\bigotimes$ Life sciences

Behavioural \& social sciences

For a reference copy of the document with all sections, see nature.com/authors/policies/ReportingSummary-flat.pdf

\section{Life sciences}

\section{Study design}

All studies must disclose on these points even when the disclosure is negative.

Sample size

Sample size was initially determined based on the number of successful plants harbouring the target mutation. From this point, growth measurements and molecules determinations were based on statistically relevant number of repetitions for ANOVA (please see materials and methods and figure legends).

Data exclusions

Replication

Randomization

Blinding
No data were excluded during growth analysis and analysis of carotenoid content.

As usual in growth measurements of plants, they were grown in controlled environmental conditions and experiments were performed in different and independent group of plants.

Two sets of plants were used depending on the genes targeted in each vector. The growth parameters and carotenoid determination were performed independently in these two set of plants.

Data were collected in randomized samples of plants size, organ length and height and tissues for carotenoid quantification

\section{Materials \& experimental systems}

Policy information about availability of materials
$\mathrm{n} / \mathrm{a}$ Involved in the study
$\square \bigotimes$ Unique materials
Х $\square$ Antibodies
Х $\square$ Eukaryotic cell lines
Х Research animals
Х $\square$ Human research participants

Unique materials

Obtaining unique materials

There are no restrictions on availability of unique materials. Both transformation vectors used and CRISPR/Cas9 plants obtained are available for research use, upon request.

\section{Method-specific reporting}

$\mathrm{n} / \mathrm{a}$ Involved in the study

X Chip-seq

Х $\square$ Flow cytometry

X $\square$ Magnetic resonance imaging 